\title{
Acute coronary syndrome in Australia: Where are we now and where are we going?
}

\author{
James Nadel, Timothy Hewitt, Damien Horton
}

University of Notre Dame Australia, Sydney

\section{REVIEW}

Please cite this paper as: Nadel J, Hewitt T, Horton D. Acute coronary syndrome in Australia: Where are we now and where are we going? AMJ 2014, 7, 3, 149-156 http://doi.org/10.21767/AMJ.2014.1921

\section{Corresponding Author:}

James Nadel

65 Hargrave St, Paddington, NSW, 2021

james.r.nadel@gmail.com

\section{ABSTRACT}

\section{Background}

Acute coronary syndrome (ACS) is a significant contributor to both morbidity and mortality in Australia. Generally speaking, sufferers of ACS who live in rural areas and are treated at rural hospitals have poorer outcomes than those living in metropolitan areas.

\section{Aims}

To characterise the differences in the management and outcomes of rural and metropolitan populations in the context of ACS, as well as identify factors responsible for these differences and suggest how they may be addressed.

\section{Method}

A review of the current literature surrounding ACS in Australia was undertaken. Through the MEDLINE/PubMed database a thorough search using the terms "acute coronary syndrome" and "Australia" identified 460 papers for review, excluding abstracts and adding "rural", "metropolitan", "reperfusion", and "outcomes" to this search narrowed the results to 149 papers for review. Data was also extracted from the Australian Institute of Health and Welfare and other Australian government publications. The review draws on insights from both local and international resources and seeks to provide an understanding of the contemporary landscape of ACS in both rural and metropolitan Australia.
The review is broken down into three key sections:

1. An outline of the 2011 National Heart Foundation of Australia/Cardiac Society of Australia and New Zealand (NHF/CSANZ) guidelines and adjuvant tools used in the assessment and treatment of ACS, and to what extent these guidelines have been implemented clinically.

2. An exploration of the current landscape of ACS in Australia and identification of the disparities facing rural populations compared to those in metropolitan areas.

3. Discussion of the factors that are resulting in poorer outcomes for ACS sufferers and suggestions of novel approaches towards addressing these factors.

\section{Conclusion}

Disparities exist between the management and outcomes of rural and metropolitan populations experiencing ACS. While the causes of these discrepancies are multifactorial; the onus is on the healthcare system to effectively reduce associated morbidity and mortality. Improvements in the management of ACS may be achieved through a continued reduction in call-to-needles time via the use of remote and mobile thrombolysis services as well as improvements in in-hospital risk assessment in order to flag and investigate those at risk of ACS.

\section{Key Words}

ACS, rural, metropolitan, Australia, outcomes

What this review adds:

\section{What is known about this subject?}

ACS contributes extensively to morbidity and mortality in Australia. Metropolitan inhabitants generally have better outcomes than those living in rural areas.

\section{What new information is offered in this study?}

This review offers insight into the differences in the management of ACS in rural versus metropolitan areas and explores the differences in outcomes for these 
population sub-groups. It also identifies causal factors for these differences and suggests ways of addressing the disparity.

3. What are the implications for research, policy, or practice? This review suggests that improvements in the management of ACS may be achieved through a continued reduction in callto-needle time via the use of remote and mobile thrombolysis services. It also encourages the implementation of in-hospital risk assessment scales to assess those at risk of recurrent ACS and the implementation of evidence-driven pharmacotherapies.

\section{Introduction}

The purpose of this paper is to better understand the disparity facing rural and metropolitan populations with Acute Coronary Syndrome (ACS) in Australia. The paper is divided into three sections: 1 ) a review of the current guidelines and other tools used in the assessment of ACS, and a snapshot of how these have been incorporated clinically in Australia; 2) a comparison of the treatment modalities and clinical outcomes of metropolitan and rural populations; and 3) a discussion of factors affecting these outcomes and reasonable approaches to rectify the inequalities faced by rural populations.

\section{Guidelines, adjuvant tools and the Australian experience of ACS in clinical practice}

The current guidelines for the management of ACS were established by the National Heart Foundation of Australia/Cardiac Society of Australia and New Zealand (NHF/CSANZ) in 2006 and have since undergone two revisions in 2007 and 2011. ${ }^{1,2}$ While the guidelines have largely remained unchanged, the addendums document the results of recently published clinical trials relevant to the treatment of ACS and describe new recommendations in light of these findings.

The guidelines are structured around the stratification of ACS into ST-elevation myocardial infarction (STEMI) or Non-STelevation Acute Coronary Syndrome (NSTEACS) with the latter encompassing both Non-STEMI and the angina spectrum. NSTEACS can be further stratified into risk groups: high (NSTEACS-HR), intermediate (NSTEACS-IR), and low (NSTEACS$L R)$ risk. A STEMI is defined as either a persistent ST-elevation $\geq 1 \mathrm{~mm}$ in two contiguous limb leads; an ST elevation $\geq 2 \mathrm{~mm}$ in two contiguous chest leads; or a new left bundle branch block pattern. Treatment recommendations for STEMI favour Percutaneous Coronary Intervention (PCl) over thrombolysis where available within 0-12 hours of symptom onset. Treatment of NSTEACS is variable depending on troponin levels at presentation and six hours after presentation and patient risk group. Nevertheless, for all patients with
NSTEACS, upgraded medical therapy and cardiac review or coronary cardiac unit (CCU) admission is advised. In all but the NSTEAC-IR with a negative stress test and NSTEAC-LR, angiography with a view to $\mathrm{PCl}$ or coronary artery bypass graft (CABG) is recommended in the subacute setting. $^{2}$

Risk stratification evidently plays a key role in assessing a patient with NSTEACS, and the NHF/CSANZ guidelines do identify specific requirements for each risk group. However, due to its emergent nature, the guidelines do not identify risk stratification for those with STEMI, where the focus is on determining the need for reperfusion rather than assessing risk. From a pragmatic point of view this presents some difficulties in establishing a comprehensive clinical approach to ACS, as accurate risk assessment could play an integral role in triage, management, and prognostication. For example, as recent studies have suggested, risk stratification may be used to identify those patients who require $\mathrm{CCU}$ admission - which has historically been based on criteria for reperfusion therapy rather than absolute risk-and in turn improve both the long and short-term clinical outcomes of ACS sufferers. ${ }^{3}$

Many clinical tools exist for risk stratification in ACS. The GRACE risk prediction tool is one such adjuvant that has been shown to be an excellent predictor of death, myocardial infarction and cumulative six-month risk of death in patients with ACS. $^{3}$ It can be used to facilitate triage and management of patients with NSTEACS as well as provide prognostication in STEMI. Born out of the ongoing global registry of acute coronary events (GRACE) - a prospective observational study involving, at the time, 94 hospitals across 14 countries, including Australia, with 43,810 patients-GRACE has been used to assess myriad outcomes in ACS. ${ }^{4}$ The GRACE risk model uses age, heart rate, systolic blood pressure, creatinine, Killip Class of cardiac heart failure, and ACS presentation factors to establish risk and can be used in conjunction with the NHF/CSANZ guidelines to direct treatment in ACS. $^{3}$

Despite having these well-established guidelines and clinically proven adjuvants for the assessment and management of ACS in Australia, recent studies have identified an "evidence-practice gap" in the clinical setting. ${ }^{5}$ Although limited, local data documenting ACS management has mimicked international trends, with many patients remaining undertreated due to limited access to $\mathrm{PCl}$ and suboptimal pharmacotherapies. ${ }^{6,7}$

One of the few available papers looking at the national management of ACS in both rural and metropolitan 
settings used the recent $A C A C I A$ registry-a nationwide prospective audit of ACS in Australia that enrolled a cohort of 3,042 patients across 39 hospitals. ${ }^{5}$ The authors identified varying compliance with guideline-recommended pharmacotherapies, reporting higher rates of aspirin and statin use; moderate rates of clopidogrel, beta-blocker and ACE-inhibitor/angiotensin receptor antagonist use; and low rates of glycoprotein Ilb/IIla inhibitor use. Regarding invasive management, the study found suboptimal rates of reperfusion therapy for STEMI across both rural and metropolitan populations. However, perhaps most disconcerting were the relatively low rates of early invasive management for NSTEACS-HR sufferers. ${ }^{5}$ Treatment of these high-risk patients is paramount in an effort to combat disease progression and symptomatic presentation and thereby reduce the enormous burden-of-care in terms of morbidity, mortality, and cost of ACS. ${ }^{8}$ While it appears that adherence to clinical guidelines is highest among STEMI sufferers, only half of NSTEACS-HR patients underwent guideline-recommended invasive management. ${ }^{5}$ This is lower than rates found in other international registries, reflecting not only patient access and preference for treatment, but also lenient risk stratification by decision-making teams. ${ }^{9}$ This snapshot of the experience of ACS in Australia highlights an inability to marry current ACS guidelines with risk assessment tools in the clinical approach to ACS.

The importance of time in ACS is another factor that has come under some scrutiny in the Australian clinical setting. Although, the advantage of $\mathrm{PCl}$ over thrombolysis has been well documented, more recent research has identified that timely reperfusion, not the modality of reperfusion is associated with significant outcome benefits. ${ }^{10,11}$ Although many definitions exist, we have understood "timely reperfusion" to mean those receiving thrombolysis within 30 minutes of presentation to first medical contact or $\mathrm{PCl}$ within 90 minutes of presentation to first medical contact. ${ }^{12}$ With data again extrapolated from the ACACIA registry, a recent paper has identified that timely reperfusion was associated with a 78 per cent reduction in mortality; nevertheless, only 23.1 per cent of STEMI patients received timely reperfusion, and only 66.9 per cent of STEMI patients received any reperfusion at all. ${ }^{11}$ Moreover, a state-wide study looking at call-to-needle (CTN) time-the time period between first notification by the patient and commencement of therapyacross Victoria, reported that only 61 per cent of patients received thrombolysis within the benchmark CTN time of 90 minutes and a CTN time of $>90$ minutes was associated with greater mortality (relative risk, 1.8). ${ }^{13}$ Hence, it appears that despite having well-established and specific guidelines regarding the treatment of STEMI, national use of timely reperfusion remains poor and incomplete in practice.
Understanding ACS guidelines and the extent to which they are followed in Australia is essential in contextualising the next section of this paper, which considers the differences in the management and outcomes of ACS among Australia's rural and metropolitan populations.

\section{Treatment modalities and clinical outcomes of metropolitan and rural populations}

ACS is a significant contributor to both morbidity and mortality in Australia, responsible for approximately 75,000 hospitalisations and 10,000 deaths every year, with this figure projected to increase to 13,675 by $2020 .^{14}$ The spectrum of ACS represents the culmination of a disease process underscored by a list of well-documented pre-event risk factors, including hypertension, smoking, and diabetes. Often overlooked are post-event risk factors that contribute to increased morbidity and mortality in patients already hospitalised with ACS, one of which is living outside of a metropolitan region.

Generally speaking, sufferers of ACS who live in rural areas and are treated at rural hospitals have poorer outcomes than those living in metropolitan areas, making rurality a major risk factor for increased post-event morbidity and mortality. ${ }^{15,16}$ Death rates from coronary heart disease (CHD) are currently reported to be 10 per cent higher in regional areas and 15 per cent higher in remote areas than in major cities, ${ }^{17}$ and various studies have highlighted the discrepancy between rural and metropolitan regions. Sexton and Sexton performed a descriptive analysis of data of 30-69-year-old Australians who died between 1986-1996 and found that, although deaths from coronary heart disease (CHD) declined overall in this period, mortality rates were up to 30 per cent higher in men and 21 per cent higher in women living outside capital cities. ${ }^{18} \mathrm{Vu}$ et al. compared in-hospital mortality for acute myocardial infarction (AMI) between metropolitan and non-metropolitan hospitals in 1,665 patients and found that the odds of 7-day mortality in non-metropolitan hospitals was significantly higher than in metropolitan hospitals. ${ }^{16}$ Beard and colleagues assessed mortality due to AMI and hospital admissions for ACS by postal area for NSW residents from 1996-2002, finding that higher levels of socioeconomic disadvantage-particularly in areas outside of major cities-were associated with increased risks of both ACS admissions and AMI mortality. ${ }^{19}$

Many studies have convincingly shown an inverse relationship between social class and $\mathrm{CHD}^{20}$ and recent research both internationally and in Australia has shown 
that living in a region of socioeconomic disadvantage increases the probability of premature death over and above the socioeconomic characteristics of the citizens of that region. ${ }^{21,22}$ There is some temptation, therefore, to attribute the rural-metropolitan outcome gap for ACS wholly to socioeconomic status. However, mortality rates for $\mathrm{CHD}$ in Australia vary geographically, even after accounting for socioeconomic disadvantage. ${ }^{23}$ Rurality itself is associated with remoteness from services, decreasing use of health services, ${ }^{24}$ and an increased proportional representation of Indigenous people, in whom ACS is particularly prevalent. ${ }^{23,25}$ It is evident then that factors beyond socioeconomic status account for the apparent outcome gap that we see between rural and metropolitan settings, many of which are further discussed in the final section of this paper. Here we will continue to address the differences in treatment modalities for ACS between rural and metropolitan settings, as we believe that the inconsistencies and inadequacies in this area can be extrapolated to account for many of the poorer outcomes that are seen.

Ultimately, the time to reperfusion is critical in the acute treatment of ACS, but for optimal management, survivors also require continuing access to specific health services such as, among other things, a cardiologist, a pharmacist, and cardiac rehabilitation facilities. ${ }^{5}$ Unfortunately, this is not always possible. As we have already seen in this paper, evidencebased guidelines are available for the management of ACS, but many inconsistencies exist in their delivery. The effective implementation of these guidelines is highly dependent upon the hospital to which the patient presents, as well as geographical location and the availability of adequate healthcare facilities in the patient's community. ${ }^{26}$ The ARIA project used GPS technology to provide a geographical model of access to reperfusion facilities by the Australian population. ${ }^{27}$ It found that 71 per cent of Australians live within an hour by road of a principal referral hospital with a cardiac catheterisation laboratory and to all aftercare services, meaning almost one-third of Australians are not able to access $\mathrm{PCl}$ within the time frames recommended in the guidelines. Similarly, it recognises that very few specialist cardiac services exist in rural and remote areas, meaning that a great deal of healthcare provision is left to the local GP.

The SNAPSHOT study specifically identifies that treatment inadequacies are more likely to occur outside of major hospitals: "Guideline-recommended investigations and therapies were provided less frequently to patients presenting to non-principal referral hospitals, regardless of patient transfers". ${ }^{28}$ Previous studies have lent weight to this assertion and have highlighted differences both in the medication and procedural domains. Using data collected from 1,400 patients aged 25-69 admitted to hospital in the lower Hunter region in NSW, Lim, O'Connell, and Heller found that patients admitted to metropolitan hospitals with AMI had shorter lengths of admission and greater use of beneficial medications (e.g., aspirin, ACE-inhibitors) compared with patients admitted to regional hospitals. ${ }^{29}$ Likewise, $\mathrm{Vu}$ et al. reported a difference in the prescription and use of beneficial cardiac medications between rural and metropolitan areas and found that the mortality difference between these two areas became negligible after adjustment for these medications was made. ${ }^{16}$ Although medical thrombolysis is available for administration in all hospitals, availability of $\mathrm{PCl}$ is much more limited, with less than 10 per cent of emergency departments in Australia located in hospitals with cardiac catheterisation facilities, and even fewer in hospitals able to perform immediate $\mathrm{PCl}^{30}$ Using data from the NSW Acute Cardiac Care Project in 1996, Heller and colleagues identified higher rates of most cardiac procedures in metropolitan hospitals than in non-metropolitan hospitals for ACS patients. ${ }^{30}$ Similarly, Beard and colleagues reported that after accounting for socioeconomic disadvantage, residents of Sydney had both a higher admission and intervention rate for ACS than residents elsewhere in NSW. ${ }^{19}$ These findings reflect, at least partially, variations in access to health services, particularly the uptake of interventions such as revascularisation procedures.

Unfortunately, there is limited data comparing rural and metropolitan outcomes from the past few years. The approach to cardiac reperfusion is a rapidly changing landscape, and newer cardiac drugs are emerging each year. Further research into how these changes are affecting the discrepancies between rural and metropolitan outcomes is strongly encouraged.

\section{Approaches to improving outcomes}

Rural populations are known to have lower socioeconomic status than people in metropolitan areas. Lower levels of income, education, and literacy rates as well as higher unemployment all contribute to the generally higher mortality rates of these populations. ${ }^{32}$ This has been shown to be no different for ACS sufferers, where it has been found that socioeconomic disadvantage increases the risk of ACS and related mortality largely due to inappropriate or inadequate management. ${ }^{19}$ Compounding the difficulty of this social gradient in health is the greater population proportion of Indigenous Australians residing in rural and remote areas. Indigenous residents have generally poorer access to healthcare services, poorer nutrition, other lifestyle factors (including higher rates of alcohol consumption and smoking), and 


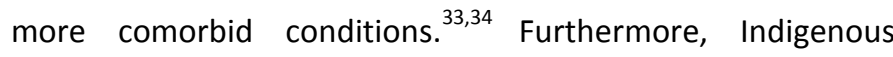
Australians have been shown to take longer to present to medical aid. ${ }^{33}$ Consequently, they tend to have poorer health outcomes than non-Indigenous Australians. ${ }^{32}$ Specifically, CHD accounts for 14 per cent of the gap in the burden of disease; ${ }^{35}$ with the age-adjusted mortality rate following $\mathrm{AMI}$ approximately 1.5 times higher for Indigenous people compared with non-Indigenous Australians. ${ }^{36}$ Limited data comparing the specific outcomes of Indigenous and nonIndigenous populations in the rural setting makes it difficult to assess the severity of the discrepancies facing these geographic sub-groups; however, Indigenous males in outer regional and remote areas have been noted to be of significantly greater risk of mortality than their counterparts because of a lack of reporting of coronary heart disease. ${ }^{37}$

Moreover, rural populations have generally lower rates of GP consultation, inadequate access to specialist services, and must travel greater distances in order to access appropriate health services. ${ }^{38,39}$ Reports by the Australian Institute of Health and Welfare (AIHW) highlight the disparity in services available to rural and remote populations compared to the metropolitan settings. ${ }^{32}$ To try to meet the demand for services in these areas, clinicians are required to work longer hours, leaving many patients unable to access busy practices in a timely manner. ${ }^{32}$ In addition, attending a general practice is prohibitively expensive for many people in rural Australia, with lower rates of bulk billing reported throughout these areas compared with metropolitan areas. ${ }^{40}$ These factors have a major impact on the management of many chronic diseases underlying $\mathrm{CHD}$ and $\mathrm{ACS}$, including regular follow-up of diabetic patients and the prescription of medications for blood pressure and cholesterol management, and thereby identifies a failure in primary and secondary prevention of this disease process. ${ }^{32}$ Attention needs to be given to the affordability and accessibility of GP services for the effective prescribing of pharmacotherapy to patients and adequate surveillance of ACS patients once they are discharged from hospital.

As mentioned earlier, geographical accessibility is a major contributing factor to the poorer outcomes of ACS in rural populations. ${ }^{32}$ An audit of findings from the ACACIA registry found that the greatest overall reduction in mortality was seen with a reduction in time for reperfusion. Nonetheless, the ACACIA registry interestingly found no statistical difference in the time to reperfusion when comparing rural and metropolitan patients. ${ }^{11}$ Recent recommendations from the NHF have led some to suggest that GP thrombolytic therapy should be explored in areas beyond the reach of hospital services. ${ }^{41}$ This notion has been supported by recent studies indicating that timely thrombolysis by GP-led emergency departments has similar results to thrombolytic therapy administered by specialist services. ${ }^{42}$ These early initiatives have been found to be effective with significant improvements in outcomes in patients presenting with STEMI. ${ }^{43}$ They present us with a pragmatic approach to reducing CTN time in ACS and eventually to potentially closing the gap between rural and metropolitan populations' access to reperfusion.

Lack of education regarding health practices and services is a frequently cited barrier to improving overall health outcomes in rural areas, and this may be responsible for the evidence suggesting that rural populations are less likely, or at the very least, delayed in seeking medical help when ACS symptoms arise. ${ }^{44}$ In the context of ACS, however, improving education may not prove to be particularly efficacious. Recent studies, including two randomised controlled trials, have highlighted that knowledge of ACS is not enough to cause a patient to seek medical attention, and that education and counselling intervention do not lead to a reduction in time from onset of symptoms to arrival at the hospital. ${ }^{45-47}$ Although advisable, it therefore seems improbable that improving health education in rural areas will alter prognosis and mortality for ACS sufferers. ${ }^{47}$

Novel initiatives to allow earlier patient management and reperfusion that have been shown to improve prognosis and mortality in patients presenting with ACS include mobile thrombolysis units, which have been used throughout Europe for many years. ${ }^{48}$ While Australia's geographical size may make this approach prohibitive for certain remote areas, this pre-hospital therapy reduces the ischaemic time of the myocardial tissue by decreasing therapy time from first symptoms and may be effective for both rural and metropolitan populations. This form of therapy, when appropriately administered by trained physicians has been shown to decrease CTN time and in turn may improve outcomes for ACS patients. ${ }^{49-50}$ Moreover, the use of these types of therapies by trained staff using well-established protocols could help in regions where travel time to thrombolysis facilities are lengthy. In these regions it could significantly reduce the time at which reperfusion therapy is commenced and thus contribute to improving outcomes and prognosis for ACS sufferers. ${ }^{51}$ Within Australia, a pilot study into prehospital thrombolysis was undertaken in 2008, but was abandoned due to poor recruitment and improved CTN time through regional thrombolysis. ${ }^{52}$ Despite its pitfalls, this study highlighted that the use of pre-hospital thrombolysis, undertaken by trained staff, could be an effective strategy in the Australian environment. Mobile thrombolysis may prove to be an interesting area for future studies into ACS. 
Despite having well-established guidelines in the management of ACS in Australia, there is still scope for improvement once patients present to medical facilities in both urban and rural settings. Improvements to services must occur to ensure adequate use of evidence-based management. As has been already identified, improved assessment is integral for the determination of treatment and assessing risk. ${ }^{3}$ Tools in risk stratification, like the GRACE risk model could help guide clinicians in triage, prognostication, and management of patients with $\mathrm{ACS}^{3-4}$ This improvement to in-hospital assessment could consequently reduce morbidity and mortality in ACS patients. Another concern has been raised regarding the management of NSTEACS-HR and IR. These patients have been shown to have inadequate rates of early invasive management, leading to an increased likelihood of an acute coronary event in a 6-month period following their primary ACS. ${ }^{5-28}$ Failure to stratify risk in these patients has been implicated as the cause of this poorer outcome. ${ }^{3-4}$ The existing risk stratification tools may be useful in increasing early treatment of patients, resulting in improved outcomes in NSTEACS. Use of these tools in clinical trials on patients presenting with ACS is advised to assess their usefulness in treatment, particularly in the NSTEACS-HR and IR groups. Other services following patient discharge from hospital also need to improve. In particular, the maximisation of effective evidence-based pharmacotherapy is vital for reducing mortality and morbidity following an ACS in at-risk patients.

\section{Conclusion}

Disparities exist between the management and outcomes of rural and metropolitan populations experiencing ACS. While the causes of these discrepancies are multifactorial, in order to effectively reduce associated morbidity and mortality the onus falls to the healthcare system. Improvements in the management of ACS may be achieved through a continued reduction in call-to-needles time via the use of remote and mobile thrombolysis services as well as improvements in inhospital risk assessment in order to flag and investigate those at risk of ACS as well as effective pharmacotherapy post-ACS.

\section{References}

1. Aroney CN, Aylward P, Chew DP, Huang N, Kelly A, White $\mathrm{H}$, et al. 2007 addendum to the National Heart Foundation of Australia/Cardiac Society of Australia and New Zealand Guidelines for the management of acute coronary syndromes 2006. Med J Aust. 2008;188(5):302.

2. Chew DP, Kelly A-M, White HD. 2011 Addendum to the national heart foundation of Australia/cardiac society of Australia and New Zealand guidelines for the management of acute coronary syndromes (ACS) 2006. Clin Trials. 2011;4:6.
3. Fox KA, Dabbous OH, Goldberg RJ, Pieper KS, Eagle KA, Van de Werf $F$, et al. Prediction of risk of death and myocardial infarction in the six months after presentation with acute coronary syndrome: prospective multinational observational study (GRACE). BMJ. 2006;333(7578):1091.

4. Goldberg RJ, Currie K, White K, Brieger D, Steg PG, Goodman SG, et al. Six-month outcomes in a multinational registry of patients hospitalized with an acute coronary syndrome (the Global Registry of Acute Coronary Events [GRACE]). Am J Cardiol. 2004;93(3):288-93.

5. Chew D, Amerena J, Coverdale S, Rankin J, Astley C, Brieger D. Current management of acute coronary syndromes in Australia: observations from the acute coronary syndromes prospective audit. Inten Med J. 2007;37(11):741-8.

6. Bhatt DL, Roe MT, Peterson ED, Li Y, Chen AY, Harrington RA, et al. Utilization of early invasive management strategies for high-risk patients with non-ST-segment elevation acute coronary syndromes. JAMA. 2004;292(17):2096-2104.

7. Eagle KA, Goodman SG, Avezum Á, Budaj A, Sullivan CM, López-Sendón J. Practice variation and missed opportunities for reperfusion in ST-segmentelevation myocardial infarction: findings from the Global Registry of Acute Coronary Events (GRACE). Lancet. 2002;359(9304):373-7.

8. AlHW. Heart, stroke and vascular disease - Australian Facts 2004. Canberra: Australian Institute of Health and Welfare; 2004.

9. Carruthers KF, Dabbous OH, Flather MD, Starkey I, Jacob A, Macleod D, et al. Contemporary management of acute coronary syndromes: does the practice match the evidence? The global registry of acute coronary events (GRACE). Heart. 2005;91(3):290-28.

10. Keeley EC, Boura JA, Grines CL. Primary angioplasty versus intravenous thrombolytic therapy for acute myocardial infarction: a quantitative review of 23 randomised trials. Lancet. 2003;361(9351):13-20.

11. Huynh LT, Rankin JM, Tideman P, Brieger DB, Erickson $M$, Markwick AJ, et al. Reperfusion therapy in the acute management of ST-segment-elevation myocardial infarction in Australia: findings from the ACACIA registry. Med J Aust. 2010;193(9):496-501.

12. Tierney W, Fitzgerald J, McHenry R. Guidelines for the management of acute coronary syndromes 2006. Med J Aust. 2006;184(8):1-32.

13. Kelly A-M, Kerr D, Patrick I, Walker T. Call-to-needle times for thrombolysis in acute myocardial infarction in Victoria. Med J Aust. 2003;178(8):381-5. 
14. AlHW. Monitoring acute coronary syndrome using national hospital data. Canberra: Australian Institute of Health and Welfare; 2011.

15. Hand R, Klemka-Walden L, Inczauskis D. Rural hospital mortality for myocardial infarction in Medicare patients in Illinois. Am J Med Qual. 1996;11(3):135-41.

16. Vu HD, Heller RF, Lim LL, D'Este C, O'Connell RL. Mortality after acute myocardial infarction is lower in metropolitan regions than in non-metropolitan regions. J Epidemiol Commun H. 2000;54(8):590-5.

17. AlHW. Rural, regional and remote health: A study on mortality (2nd edition). Canberra: Australian Institute of Health and Welfare; 2007.

18. Sexton PT, Sexton TL. Excess coronary mortality among Australian men and women living outside the capital city statistical divisions. Med J Aust. 2000;172(8):370-4.

19. Beard JR, Earnest A, Morgan G, Chan H, Summerhayes R, Dunn TM, et al. Socioeconomic disadvantage and acute coronary events: a spatiotemporal analysis. Epidemiology. 2008;19(3):485-92.

20. González MA, Artalejo FR, del Rey Calero J. Relationship between socioeconomic status and ischaemic heart disease in cohort and case-control studies: 1960-1993. Int J Epidemiol. 1998;27(3):350-8.

21. Turrell G, Kavanagh A, Draper G, Subramanian S. Do places affect the probability of death in Australia? A multilevel study of area-level disadvantage, individuallevel socioeconomic position and all-cause mortality, 1998-2000. J Epidemiol Commun H. 2007;61(1):13-19.

22. Pickett KE, Pearl M. Multilevel analyses of neighbourhood socioeconomic context and health outcomes: a critical review. J Epidemiol Commun H. 2001;55(2):111-122.

23. Burnley $\mathrm{IH}$. Transitions and inequalities in acute myocardial infarction mortality in New South Wales, 1969-94. Aust NZ J Publ Heal. 1999;23(4):343-351.

24. Eckert KA, Taylor AW, Wilkinson D. Does health service utilisation vary by remoteness? South Australian population data and the Accessibility and Remoteness Index of Australia. Aust NZ J Publ Heal. 2004;28(5):426432.

25. Randall DA, Jorm LR, Lujic S, O'Loughlin AJ, Churches TR, Haines $\mathrm{MM}$, et al. Mortality after admission for acute myocardial infarction in Aboriginal and non-Aboriginal people in New South Wales, Australia: a multilevel data linkage study. BMC Public Health. 2012;12:281.

26. Baldwin L-M, Chan L, Andrilla CHA, Huff ED, Hart LG. Quality of care for myocardial infarction in rural and urban hospitals. J Rural Health. 2010;26(1):51-7.

27. Clark RA, Coffee N, Turner D, Eckert KA, van Gaans D, Wilkinson $D$, et al. Application of geographic modeling techniques to quantify spatial access to health services before and after an acute cardiac event: the Cardiac
Accessibility and Remoteness Index for Australia (ARIA) project. Circulation. 2012;125(16):2006-14.

28. Chew DP, French J, Briffa TG, Hammett CJ, Ellis CJ, Ranasinghe I, et al. Acute coronary syndrome care across Australia and New Zealand: the SNAPSHOT ACS study. Med J Aust. 2013;199:1-7.

29. Lim LL, O'Connell RL, Heller RF. Differences in management of heart attack patients between metropolitan and regional hospitals in the Hunter Region of Australia. Aust NZ J Publ Heal. 1999;23(1):61-6.

30. AlHW. Australian hospital statistics 2011-2012. Canberra: Australian Insititute of Health and Welfare; 2013.

31. Heller RF, O'Connell RL, D'Este C, Lim LL, Fletcher PJ. Differences in cardiac procedures among patients in metropolitan and non-metropolitan hospitals in New South Wales after acute myocardial infarction and angina. The Aust J Rural Health. 2000;8(6):310-7.

32. AlHW. Rural, regional and remote health: Indicators of health status and determinants of health. Canberra: Australian Institute of Health and Welfare; 2008.

33. Australian Indigenous HealthInfoNet. Summary of Australian Indigenous Health, 2012. Perth 2013.

34. AlHW. Aboriginal and Torres Strait Islander Health Performance Framework 2012: detailed analyses Canberra: Australian Institute of Health and Welfare 2013.

35. Vos T, Barker B, Begg S, Stanley L, Lopez AD. Burden of disease and injury in Aboriginal and Torres Strait Islander Peoples: the Indigenous health gap. Int J Epidemiol. 2009;38(2):470-7.

36. Mathur S, Moon L, Leigh S. Aboriginal and Torres Strait Islander people with coronary heart disease: further perspectives on health status and treatment. Canberra: Australian Institute of Health and Welfare; 2006.

37. AlHW. Rural, regional and remote health: A study on mortality. 2nd edition. Canberra 2007.

38. Farmer J, Munoz S-A, Threlkeld G. Theory in rural health. Aust J Rural Health. 2012;20(4):185-9.

39. Jordan S, Wilson A, Dobson A. Management of heart conditions in older rural and urban Australian women. Intern Med J. 2011;41(10):722-9.

40. Young AF, Dobson AJ. The decline in bulk-billing and increase in out-of-pocket costs for general practice consultations in rural areas of Australia, 1995-2001. Med J Aust. 2003;178(3):122-126.

41. Brieger D, Kelly A-M, Aroney C, Tideman P, Freedman $S B$, Chew D, et al. Acute coronary syndromes: consensus recommendations for translating 
knowledge into action. Med J Aust. 2009;191(6):334-8.

42. Krones R, Radford P, Cunningham C, Krones D, Haines HM. Thrombolysis for acute ST elevation myocardial infarction: a pilot study comparing results from GP led small rural health emergency departments with results from a physician led sub-regional emergency department. Rural and Remote Health. 2012;12:2013.

43. Krumholz HM, Bradley $\mathrm{EH}$, Nallamothu BK, Ting $\mathrm{HH}$, Batchelor WB, Kline-Rogers $E$, et al. A Campaign to Improve the Timeliness of Primary Percutaneous Coronary Intervention Door-to-Balloon: An Alliance for Quality.J Am Coll Cardiol.2008;1(1):97-104.

44. Baker T, McCoombe S, Mercer-Grant C, Brumby S. Chest pain in rural communities; balancing decisions and distance. Emerg Med Australas. 2011;23(3):337-45.

45. Ballard N, Bairan A, Newberry L, Van Brackle L, Barnett G. Effect of education on a chest pain mnemonic on door-toECG time. J Emerg Nurs. 2011;37(3):220-4.

46. Thuresson $M$, Jarlöv MB, Lindahl B, Svensson L, Zedigh $C$, Herlitz J. Thoughts, actions, and factors associated with prehospital delay in patients with acute coronary syndrome. Heart Lung. 2007;36(6):398-409.

47. Dracup K, McKinley S, Riegel B, Moser DK, Meischke H, Doering LV, et al. A randomized clinical trial to reduce patient prehospital delay to treatment in acute coronary syndrome. Circulation. 2009;2(6):524-32.

48. Arntz H-R, Stern R, Linderer T, Schröder R. Efficiency of a physician-operated mobile intensive care unit for prehospital thrombolysis in acute myocardial infarction. Am J Cardiol. 1992;70(4):417-20.

49. Schofer J, Büttner J, Geng G, Gutschmidt K, Herden HN, Mathey DG, et al. Prehospital thrombolysis in acute myocardial infarction. Am J Cardiol. 1990;66(20):1429-33.

50. Zeymer U, Arntz H-R, Dirks B, Ellinger K, Genzwürker H, Nibbe $L$, et al. Reperfusion rate and inhospital mortality of patients with ST segment elevation myocardial infarction diagnosed already in the prehospital phase: Results of the German Prehospital Myocardial Infarction Registry (PREMIR). Resuscitation. 2009;80(4):402-6.

51. Crowder JS, Hubble MW, Gandhi S, McGinnis H, Zelman S, Bozeman $W$, et al. Prehospital administration of tenecteplase for ST-segment elevation myocardial infarction in a rural EMS system. Prehosp Emerg Care. 2011;15(4):499-505.

52. Kerr D, Jennings $P$, Kelly $A-M$, Walker $T$, Edington J. Pilot of prehospital thrombolysis in ST elevation myocardial infarction. JEPHC. 2008;6(1).

\section{PEER REVIEW}

Not commissioned. Externally peer reviewed.

\section{CONFLICTS OF INTEREST}

The authors declare that they have no competing interests.

\section{ETHICS COMMITTEE APPROVAL}

All aspects of this project have been undertaken upholding the ethical guidelines established by both the NHMRC and University of Notre Dame's HREC. 